\title{
BMJ Open Protocol for a randomised controlled trial of continuous infusions of vancomycin to improve the attainment of target vancomycin levels in young infants: The VANC trial
}

\author{
Amanda Gwee, ${ }^{1,2,3}$ Noel Cranswick, ${ }^{1,2,3}$ Susan M Donath, ${ }^{2,3}$ Rodney Hunt, ${ }^{1,2,3}$ \\ Nigel Curtis ${ }^{1,2,3}$
}

To cite: Gwee A, Cranswick N, Donath SM, et al. Protocol for a randomised controlled trial of continuous infusions of vancomycin to improve the attainment of target vancomycin levels in young infants: The VANC trial. BMJ Open 2018;8:e022603. doi:10.1136/ bmjopen-2018-022603

\section{- Prepublication history for} this paper is available online. To view these files, please visit the journal online (http://dx.doi. org/10.1136/bmjopen-2018022603).

Received 25 February 2018 Revised 14 September 2018 Accepted 17 September 2018

Check for updates

(c) Author(s) (or their employer(s)) 2018. Re-use permitted under CC BY-NC. No commercial re-use. See rights and permissions. Published by BMJ.

${ }^{1}$ Departments of General Medicine and Neonatal Medicine, The Royal Children's Hospital, Melbourne, Victoria, Australia

${ }^{2}$ Infectious Diseases \& Microbiology, Neonatal Research, Clinical Epidemiology \& Biostatistics Unit, Murdoch Children's Research Institute, Parkville, Victoria, Australia ${ }^{3}$ Department of Paediatrics, The University of Melbourne, Parkville, Victoria, Australia

Correspondence to

Dr Amanda Gwee;

amanda.gwee@rch.org.au

\section{ABSTRACT}

Introduction Vancomycin is frequently used in the treatment of late-onset sepsis in young infants and is routinely administered as intermittent infusions (IIV); however, existing IIV dosing guidelines achieve target vancomycin levels in less than half of infants. Continuous infusions of vancomycin (CIV) are an attractive alternative as adult studies report a higher attainment of target vancomycin levels, simpler drug monitoring and fewer drug side effects.

Methods This is a multicentre, randomised controlled trial in which 200 young infants (aged 0-90 days) requiring vancomycin will be randomised to CIV or IIV for a duration determined by the treating clinician. Vancomycin levels will be measured immediately after the first dose in both arms. Trough and peak levels will be determined in the IIV arm and steady-state levels 18-30 hours after commencement of infusion will be measured in the CIV arm. Full blood count, urea and electrolytes, and $C$ reactive protein level will be monitored throughout treatment. For all Grampositive bacteria isolated from blood culture, a vancomycin Etest will be done to determine the minimum inhibitory concentration of the bacterium.

Analysis Primary outcome: the proportion of infants with levels within target range at their first steady-state concentration. Secondary outcomes: (1) the proportion of drug-related adverse effects; (2) the time to achieve target levels in the blood; (3) the pharmacodynamics of vancomycin (using non-linear mixed effect modelling). Ethics and dissemination The study has been approved by The Royal Children's Hospital Melbourne Human Research Ethics Committee (HREC) (No. 34030) and the South Eastern Sydney Local Health District HREC (SSA 16/G/335). Results will be published in a peer-reviewed journal.

Trial registration number NCT02210169.

\section{INTRODUCTION}

Bloodstream infections are one of the leading causes of death in young infants worldwide. ${ }^{1}$ In developed countries, Staphylococcus aureus and coagulase-negative staphylococci (CONS) are among the most common
Strengths and limitations of this study

- Continuous infusions of vancomycin may minimise errors in drug dosing and therapeutic drug monitoring in young infants.

- First randomised controlled trial of vancomycin dosing in young infants.

- Not designed to detect difference in drug-related adverse effects.

- Unblinded study design due to different methods of administration.

pathogens causing late-onset sepsis. ${ }^{23}$ A large proportion (up to $84 \%$ in one study) of CONS isolates possess the mecA gene which confers resistance to beta-lactam antibiotics ${ }^{45}$; vancomycin is therefore an important drug in this age group. Despite the frequent use of vancomycin in young infants, there is no consensus on the optimal dosing regimen.

Traditionally, vancomycin has been administered as an intermittent infusion (IIV) with therapeutic drug monitoring (TDM) of trough levels. Multiple different IIV dosing regimens are routinely used, but all result in subtherapeutic trough levels in the majority of young infants. ${ }^{6}$ Furthermore, errors with the timing of trough sampling has been associated with incorrect dose adjustments and the need for repeated blood samples for TDM. $^{7}$ An alternative dosing regimen is to administer vancomycin as a continuous infusion (CIV) over a 24-hour period.

Vancomycin was previously thought to exhibit time-dependent bacterial killing with optimal effect achieved when the serum vancomycin level is three to four times the minimum inhibitory concentration (MIC, the lowest antibiotic concentration that will stop growth) of the bacteria. ${ }^{8}$ With other 
time-dependent killing antibiotics, such as piperacillin-tazobactam, extended infusion has been shown to improve clinical outcome. ${ }^{9}$ It has therefore been suggested that there may be the same benefit when vancomycin is administered as a continuous infusion.

Recent studies of adults with $S$. aureus infections report a strong association between improved clinical outcome and vancomycin exposure (defined as the area under the concentration-time curve (AUC)) ${ }^{10}$ As a result, a ratio of the AUC to the MIC of the bacteria causing infection exceeding 400 (AUC/MIC >400) is recommended as the vancomycin pharmacodynamic target in the Infectious Diseases Society of America guidelines. ${ }^{11}$ There are no published studies investigating the relationship between vancomycin exposure and clinical effect in infants nor is there an established pharmacodynamic target for the treatment of CONS infections. Currently in children, trough levels of $10-20 \mathrm{mg} / \mathrm{L}$ are targeted for IIV and there are limited data on the correlation between trough levels and AUC.

The existing studies in adults comparing IIV to CIV report improved attainment and maintenance of target blood levels, the avoidance of repeated line access thus reducing the risk of infections and reduction in vancomycin-associated nephrotoxicity. ${ }^{11}{ }^{12}$ These studies have reported comparable treatment outcomes to IIV and one randomised controlled trial reported a reduction in mortality with CIV. ${ }^{12}{ }^{13} \mathrm{CIV}$ also allows for safer and easier TDM: levels can simply be measured at the same time as routine blood investigations. ${ }^{7}$ This is especially important in premature infants who are prone to iatrogenic anaemia.

In five prospective cohort studies of CIV in young infants, up to $88 \%$ of blood vancomycin level measurements were within target range with CIV compared with less than $50 \%$ with IIV. ${ }^{14}$ Unfortunately, none of these studies assessed clinical outcomes such as clearance of infection or mortality although they did report that CIV was not associated with any side effects in young infants. ${ }^{15-19}$ There have been no randomised controlled trials comparing CIV to IIV dosing in infants.

Our primary objective is to directly compare CIV with IIV in a randomised controlled trial to determine which dosing regimen results in the highest proportion of patients attaining target vancomycin concentrations at the first steady-state level (when the blood vancomycin level is in equilibrium). Our secondary objectives are to compare (1) the rate of drug-related adverse effects, (2) the time taken to achieve target therapeutic vancomycin levels in blood, and (3) to determine the pharmacodynamics (the relationship between clinical parameters and adverse effects with serum levels) of vancomycin in young infants using non-linear mixed effects modelling (NONMEM).

\section{METHODS AND ANALYSIS}

\section{Study aims}

Primary aim

To compare the proportion of young infants achieving target therapeutic vancomycin levels in blood at their first steady-state level. Steady state is defined as 24-48 hours after commencement or dose change for both CIV and IIV. ${ }^{16}$

\section{Secondary aims}

To determine, for CIV and IIV in young infants:

1. The rate of drug-related adverse effects, including infusion-related side effects (red man syndrome) and renal toxicity.

2. The time taken to achieve target therapeutic vancomycin levels in blood.

3. The pharmacodynamics of vancomycin in young infants, including the relationship between clinical parameters ((i) duration of fever; (ii) clearance of bacteria from blood; (iii) the normalisation of inflammatory markers (white cell count and indices, $\mathrm{C}$ reactive protein (CRP)); (iv) duration of inotropes and (v) patient deaths) and serum vancomycin concentrations and AUC using NONMEM.

\section{Study design and setting}

This is a multicentre, unblinded, randomised controlled trial of IIV versus CIV. Clinical trials.gov NCT02210169 (Recruitment status: completed).

The study population will be young infants aged $0-90$ days in two tertiary neonatal intensive care units. Study sites comprise the Royal Children's Hospital (RCH) Melbourne, Australia and Royal Hospital for Women (RHW), Sydney, Australia. The current protocol was last updated on the 5 June 2017, V.15. RCH is the coordinating centre and RCH investigators are responsible for the coordination, data management and overseeing of the trial.

\section{Eligibility criteria}

The inclusion criteria are young infants aged between 0 and 90 days admitted to the neonatal unit with a suspected infection requiring treatment with vancomycin for 48 hours or more as determined by the treating physician. The exclusion criteria are (1) corrected gestational age $<25$ weeks; (2) allergy to any glycopeptide antibiotic; (3) vancomycin administered within the previous 72 hours; (4) prior randomisation in the study; and (5) renal impairment (as defined in the Victorian Neonatal handbook). ${ }^{20}$

\section{Interventions}

\section{Dosing protocol}

Infants will be randomised to receive CIV or IIV for a duration determined by the treating doctor. Study involvement is from the time of randomisation until vancomycin is ceased.

\section{IIV group}

Vancomycin will be administered according to standard of care, the British National Formulary vancomycin dosing regimen (see table 1). ${ }^{21}$ Doses will be infused intravenously over 1 hour. The target blood vancomycin concentration for IIV is $10-20 \mathrm{mg} / \mathrm{L}$. 


\begin{tabular}{lll}
\hline $\begin{array}{l}\text { Table } 1 \\
\text { regimen }\end{array}$ & Intermittent infusion of vancomycin empiric dosing \\
\hline $\begin{array}{l}\text { Corrected gestational } \\
\text { age in weeks }\end{array}$ & $\begin{array}{l}\text { Dose (mg/kg/ } \\
\text { dose) }\end{array}$ & $\begin{array}{l}\text { Dose interval } \\
\text { (hourly) }\end{array}$ \\
\hline$<29$ & 15 & 24 \\
\hline $29-35$ & 15 & 12 \\
$36-44$ & 15 & 8 \\
$>44$ & 15 & 6 \\
\hline
\end{tabular}

If the vancomycin level is outside the target range, the dose will be adjusted as per the protocol described in table 2. If a trough level exceeds $20 \mathrm{mg} / \mathrm{L}$, the dose will be withheld and the trough level repeated before each due dose until the level is $\leq 20 \mathrm{mg} / \mathrm{L}$. Once the vancomycin level is $\leq 20 \mathrm{mg} / \mathrm{L}$, dosing will be restarted at a lower dose frequency (see table 2 ).

\section{CIV group}

An initial intravenous loading dose of $15 \mathrm{mg} / \mathrm{kg}$ will be given over 1 hour followed by a continuous intravenous infusion of vancomycin over a 24-hour period (see table 3$)^{7}$

The target vancomycin concentration is $15-25 \mathrm{mg} / \mathrm{L}{ }^{718}$ If the concentration is outside of this range, the adjusted dose ( $\mathrm{mg} /$ day) will be calculated by: last maintenance dose $\times($ target concentration $(20 \mathrm{mg} / \mathrm{L}) /$ last vancomycin concentration).

\section{Blood sampling protocol}

The following blood samples will be collected.

- Albumin level-measured at baseline.

- Full blood examination, urea, electrolytes and creatinine, CRP level-measured at baseline, every 48 hours during vancomycin therapy for the first week, then weekly thereafter. (The CRP will only be repeated if initially elevated.)

- Blood culture-taken prior to vancomycin therapy. This will be repeated if the initial blood culture is positive. The vancomycin MIC of all isolated Gram-positive bacteria will be determined by Etest.

- Serum vancomycin concentrations will be measured in $0.5 \mathrm{~mL}$ blood samples by enzyme immunoassay at the clinical laboratories of the RCH and RHW. Samples for TDM will be taken at the time points shown in figures 1 and 2 .

Blood samples will be taken from a different line whenever practical. However, if the infant has only one line, the sample will be taken via heel prick or through the line after an appropriate discard.

\section{Reasons for withdrawal}

Reasons for withdrawal from the study will be recorded along with demographic data. These may include drug-related adverse effects, drug incompatibilities, treatment failure/death, transfer to another health facility or guardian/parent request.
Table 2 Intermittent infusion of vancomycin dose adjustment protocol

\begin{tabular}{lll}
\hline $\begin{array}{l}\text { Current } \\
\text { regimen }\end{array}$ & If trough $<\mathbf{1 0} \mathbf{m g} / \mathbf{L}$ & If trough $>\mathbf{2 0} \mathbf{m g} / \mathbf{L}$ \\
\hline 6 hourly & Change to 4 hourly & Change to 8 hourly \\
\hline 8 hourly & Change to 6 hourly & Change to 12 hourly \\
\hline 12 hourly & Change to 8 hourly & Change to 18 hourly \\
\hline 18 hourly & Change to 12 hourly & Change to 24 hourly \\
\hline 24 hourly & Change to 18 hourly & Change to 30 hourly \\
\hline
\end{tabular}

\section{Outcomes}

The primary outcome is the proportion of infants achieving target concentrations at the first steady-state level. The secondary outcomes are (1) the proportion of drug-related adverse effects with CIV compared with IIV, (2) the time taken to achieve target therapeutic vancomycin levels in blood and (3) the pharmacokinetics and pharmacodynamics of vancomycin in young infants (using NONMEM).

\section{Sample size and power calculation}

Two hundred young infants will be randomly assigned to receive CIV or IIV. This sample size is calculated to detect a difference of $20 \%$ in the proportion of patients achieving target vancomycin levels between the two groups $(50 \%$ attainment with IIV, $70 \%$ attainment with CIV) with a power of $80 \% .^{1419}$ This difference of $20 \%$ is based on previous studies reporting attainment of target concentrations with IIV ranging from $40 \%$ to $50 \%$, 192223 and our preparatory review finding attainment of target concentrations with CIV ranging from $74 \%$ to $89.2 \% .^{14} 19$ This sample size was calculated with a Pearson's $\chi^{2}$ test using Stata (2015).

\section{Recruitment}

Recruitment will involve two processes: (1) early consent will be sought from the parent/guardian of those patients who are likely to require vancomycin therapy (ie, those with surgical conditions, indwelling foreign material, colonised with bacteria requiring treatment with vancomycin). Consent will be confirmed verbally if the patient is subsequently randomised in the study. (2) Eligible patients will be identified by the clinical staff following staff education sessions. In addition, an in-built notification system

Table 3 Continuous infusions of vancomycin empiric dosing regimen (described in Patel et al) ${ }^{7}$

\section{Serum creatinine} level ( $\mu \mathrm{mol} / \mathrm{L})$

CGA (weeks)

Dose (mg/kg/day)

\begin{tabular}{lll}
\hline$<40$ & $\geq 40$ & 50 \\
$<40$ & $<40$ & 40 \\
$40-60$ & All & 30 \\
$>60$ & All & 20
\end{tabular}

CGA, corrected gestational age. 


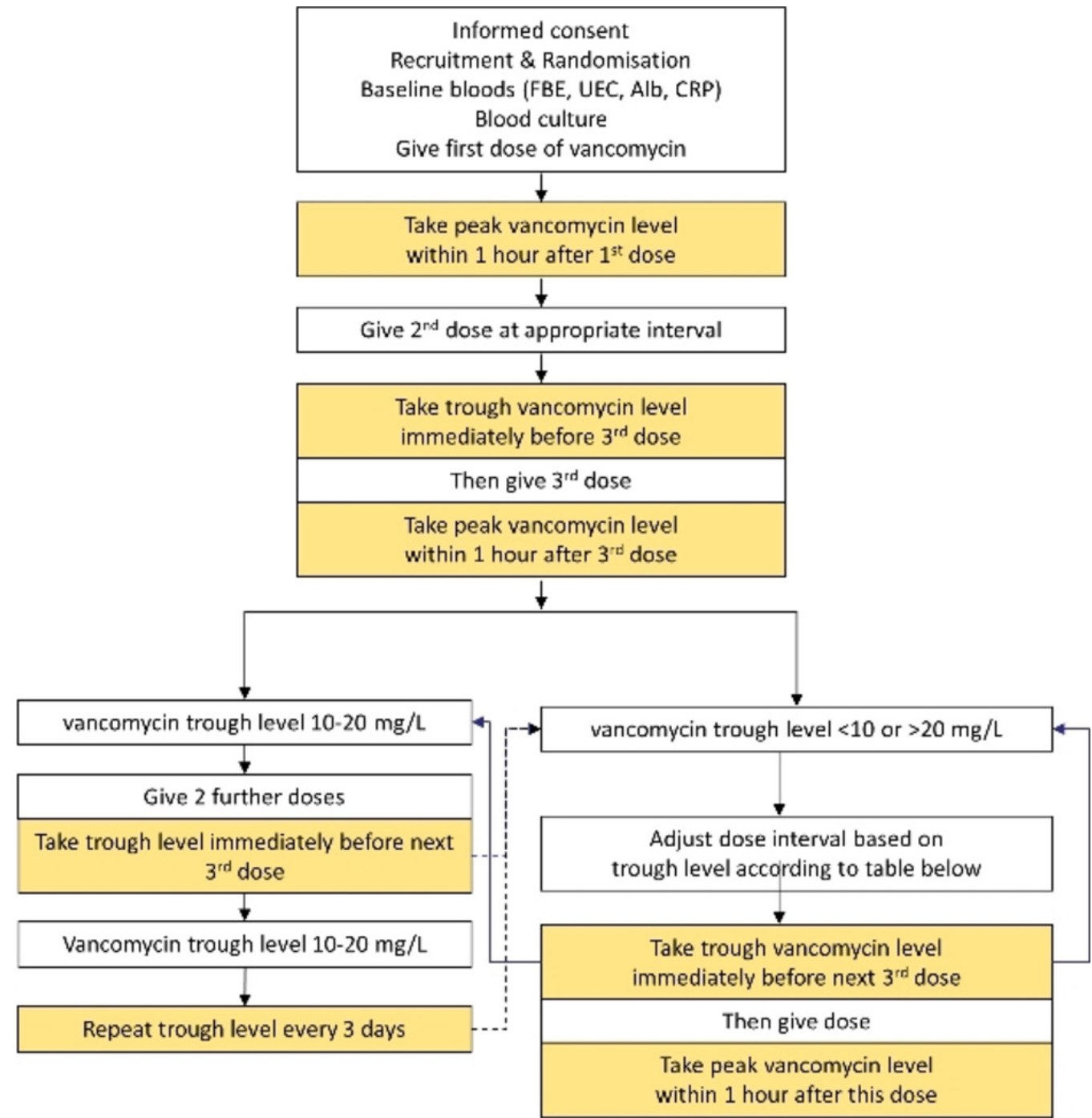

Figure 1 Intermittent infusion of vancomycin level monitoring algorithm. Alb, albumin; CRP, C reactive protein; FBE, full blood examination; UEC, urea, electrolytes and creatinine.

within the hospital electronic medical record will notify a member of the investigator team if an eligible infant is prescribed vancomycin. As the initial dose in both arms of the study is the same, investigators will have until the end of the first dose to gain consent for randomisation.

\section{Allocation}

If the treating clinician determines that an infant requires treatment with vancomycin, the patient will be randomised in a 1:1 ratio to either CIV or IIV. The randomisation schedule will be generated by a statistician external to the study who will use random permuted blocking to ensure allocation concealment. At RCH, the infusion mode will be contained in sealed opaque envelopes, labelled consecutively with the randomisation numbers. At RHW, staff will randomise the infant using an online randomisation procedure. Clinicians will not be blinded to the allocated treatment.

\section{Data collection}

Data will be entered into a password-protected online database on the Murdoch Children's Research Institute
(MCRI) server. Patient information consent forms (PICF) will be completed in hard copy. Data on patient demographics (gestational age, birth and current weight, sex, date of birth), dosing (timing and dose), laboratory data (as outlined in figures 1 and 2) and clinical details (underlying diagnosis, indication for vancomycin treatment, concomitant nephrotoxic drugs, duration of fever, requirement for inotropes, presence/absence of apnoeas) will be collected by a member of the investigative team.

\section{Data management and access to data}

Participant information will be coded by study reference number in deidentified form that will allow the data to be reidentifiable. The original data collection sheets will be stored in a locked cabinet in an area with restricted access at the MCRI. The database will only be accessible by the investigators and study coordinators. As per the Victorian guidelines, data will be disposed of when the youngest participant reaches age 25 years. 


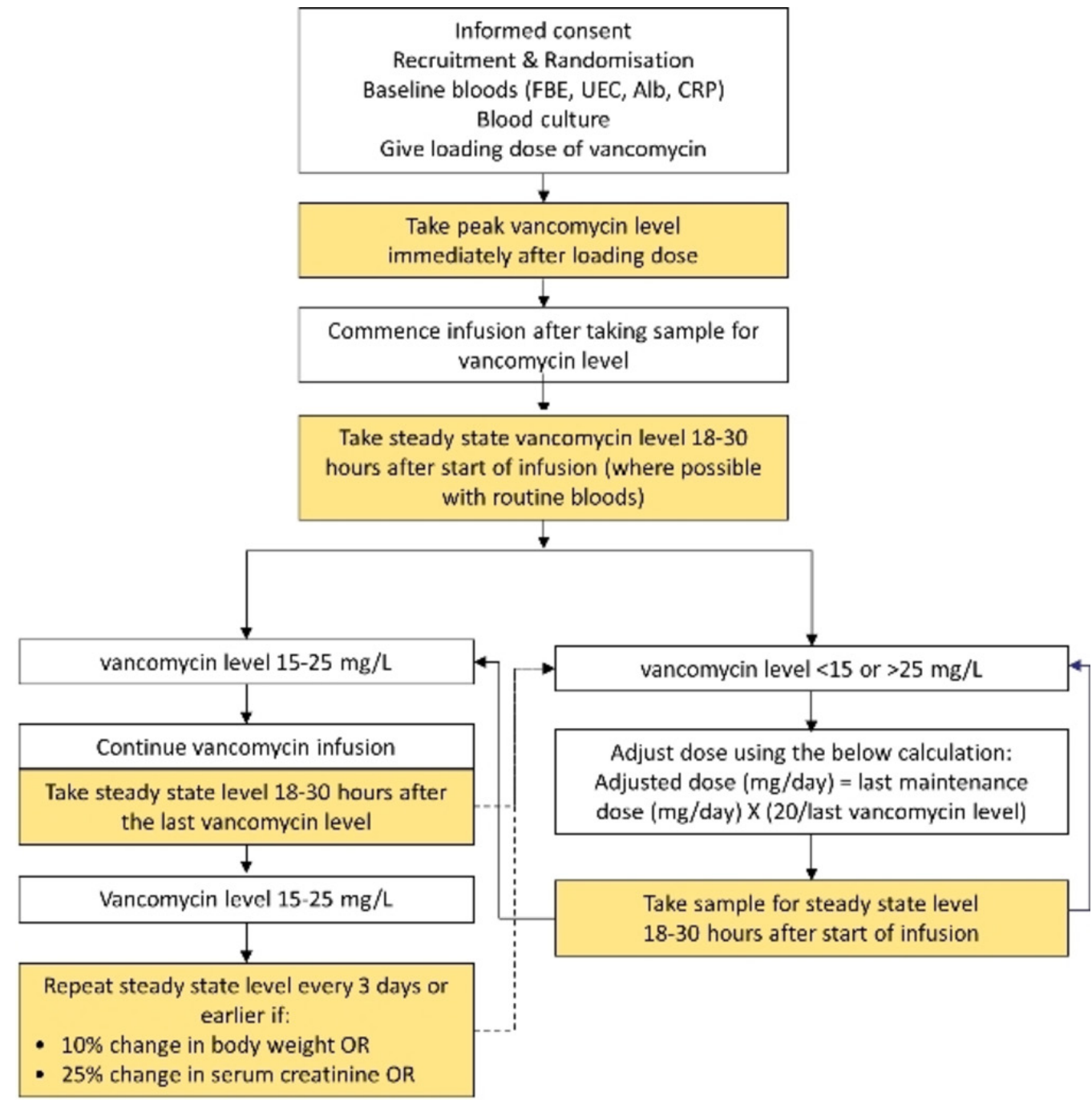

Figure 2 Continuous infusions of vancomycin level monitoring algorithm. Alb, albumin; CRP, C reactive protein; FBE, full blood examination; UEC, urea, electrolytes and creatinine.

\section{Statistical methods}

Statistical analysis will be overseen by the trial statistician. Randomised infants who had the first trough vancomycin level (IIV) or steady-state level (CIV) measured will be included in the intention-to-treat analysis. Data will be collected to meet the Consolidated Standards of Reporting Trials guidelines for reporting randomised trials. $^{24}$

\section{Primary outcome}

The difference in proportions of infants achieving target concentration at steady state will be estimated, together with a $95 \%$ CI. The null hypothesis that there is no difference in the proportions achieving target concentration will be assessed using $\chi^{2}$ test.

\section{Secondary outcomes}

All secondary outcomes that are proportions will be analysed as described above.

The time to achieve target levels will be determined by developing a population pharmacokinetic model using NONMEM. A first-order conditional estimation method will be used to estimate pharmacokinetic parameters and their variability. Model validation will be based on graphical and statistical criteria, including goodness-of-fit plots, bootstrapping, visual predictive checks and normalised prediction distribution errors. The parameter estimates obtained from the model will be used for vancomycin dosing optimisation. The concentration-time profiles of the two dosing regimens will be predicted in preterm and term infants. The site at which the sample is processed will be a variable in our population pharmacokinetic model. The pharmacodynamics of vancomycin will be evaluated using NONMEM. Subgroup analysis of the following two groups will be done: (1) those with a positive blood culture to determine the effects of bacterial species and MIC on clinical response and (2) those with the clinical syndrome of sepsis but with negative blood cultures.

\section{Data monitoring and auditing}

A data safety monitoring committee independent from the study team will meet when recruitment of 50, 100 and 
150 infants has been achieved. An interim analysis will not be performed.

\section{Harms}

The potential harms of the study include: (1) blood sampling-participants will have blood tests for vancomycin levels that are additional to routine care. However, the volume of blood per participant falls within recommended guidelines for research blood sampling in children and where possible, blood samples will be collected at the same time as clinical samples. ${ }^{25}$ (2) Poor line access with CIV prohibiting the administration of other drugs. A continuous infusion can be stopped for $60 \mathrm{~min}$ or less, three times per day if a drug that is incompatible with vancomycin is required. This will be documented in a case report form. If there is requirement for a drug that requires a prolonged infusion in the absence of other line space, the patient will be withdrawn from the study. All adverse events will be recorded, and serious adverse events notified to the human research ethics committee within 24 hours.

\section{Outlook and significance}

This is the first paediatric randomised controlled trial of CIV compared with IIV and the first to assess the pharmacodynamics of vancomycin in young infants. The results of our trial will influence national and international treatment guidelines for young infants. This will potentially enable safer and more effective use of a commonly used drug in paediatric patients worldwide.

\section{Limitations}

Potential limitations of the study design include the unblinded design of the trial that could lead to bias. However, the primary outcome is a laboratory result; therefore, blinding would not influence this. Although the CIV dose should ideally be calculated based on drug clearance, formulas for determination of creatinine clearance (eg, Cockroft-Gault formula) are unreliable in this age group. ${ }^{26}$ As a secondary outcome, we aim to assess the difference in drug-related adverse effects in the two groups; however, the frequency of side effects associated with vancomycin is low, and therefore 200 infants may not be sufficient to detect a difference between the two groups. For determination of the MIC of bacteria, although broth microdilution is the gold standard, Etest is an established and accepted method for MIC determination used in other pharmacodynamic studies. ${ }^{27}$

\section{Patient and public involvement}

This research question was developed with the aim of minimising harm to the patient by optimising vancomycin dosing and reducing the errors around vancomycin blood level monitoring. Patients were not involved in the design of the study, recruitment or conduct of the study. Study results will be available through publication in a peer-reviewed journal. The burden of the intervention was not assessed by patients.

\section{Current status}

Recruitment has commenced at both sites and is expected to complete by September 2018.

\section{ETHICS AND DISSEMINATION}

\section{Consent or assent}

A study logbook of all consents and refusals will be maintained. Parent(s)/guardian(s) will be reassured that declining to participate in the study will not affect their child's clinical care and if they consent, they have the option to withdraw at any time. The parent/guardian will complete a written PICF as part of the consent process.)

\section{Confidentiality}

All outcomes will be presented as aggregated data and no individual will be identifiable.

\section{Dissemination policy}

Outcomes of this protocol will be published in a peer-reviewed journal and incorporated into national guidelines.

Acknowledgements The authors thank the staff and families at RCH and RHW for supporting this trial.

Contributors AG, NCr and NCu developed the research question, protocol and drafted the first version of the manuscript. SMD and RH advised and assisted with protocol development and critically revised the manuscript. All authors edited, reviewed and approved the final version of this manuscript.

Funding This project is funded by a research grant from Murdoch Children's Research Institute. AG has been funded by a Royal Australasian College of Physicians Basser Research Entry Scholarship as well as a National Health and Medical Research Council postgraduate scholarship.

Disclaimer The study funders did not have authority over the study design or preparation of this report.

Competing interests None declared.

Patient consent Not required.

Ethics approval Royal Children's Hospital Melbourne and South Eastern Sydney Local Health District.

Provenance and peer review Not commissioned; externally peer reviewed.

Open access This is an open access article distributed in accordance with the Creative Commons Attribution Non Commercial (CC BY-NC 4.0) license, which permits others to distribute, remix, adapt, build upon this work non-commercially, and license their derivative works on different terms, provided the original work is properly cited, appropriate credit is given, any changes made indicated, and the use is non-commercial. See: http://creativecommons.org/licenses/by-nc/4.0/.

\section{REFERENCES}

1. Vergnano S, Sharland M, Kazembe P, et al. Neonatal sepsis: an international perspective. Arch Dis Child Fetal Neonatal Ed 2005;90:F220-4.

2. Baltimore RS. Neonatal sepsis: epidemiology and management. Paediatr Drugs 2003;5:723-40.

3. Hornik CP, Fort P, Clark RH, et al. Early and late onset sepsis in verylow-birth-weight infants from a large group of neonatal intensive care units. Early Hum Dev 2012;88:S69-74.

4. Ternes YM, Lamaro-Cardoso J, André MC, et al. Molecular epidemiology of coagulase-negative Staphylococcus carriage in neonates admitted to an intensive care unit in Brazil. BMC Infect Dis 2013;13:572.

5. Brzychczy-Wloch M, Borszewska-Kornacka M, Gulczynska E, et al. Prevalence of antibiotic resistance in multi-drug resistant coagulasenegative staphylococci isolated from invasive infection in very low birth weight neonates in two Polish NICUs. Ann Clin Microbiol Antimicrob 2013;12:41. 
6. Vandendriessche A, Allegaert K, Cossey V, et al. Prospective validation of neonatal vancomycin dosing regimens is urgently needed. Curr Ther Res Clin Exp 2014;76:51-7.

7. Patel $A D$, Anand $D$, Lucas $C$, et al. Continuous infusion of vancomycin in neonates. Arch Dis Child 2013;98:478-9.

8. Foster JK, Lentino JR, Strodtman R, et al. Comparison of in vitro activity of quinolone antibiotics and vancomycin against gentamicinand methicillin-resistant staphylococcus aureus by time-kill kinetic studies. Antimicrob Agents Chemother 1986;30:823-7.

9. Lodise TP, Lomaestro B, Drusano GL. Piperacillin-tazobactam for Pseudomonas aeruginosa infection: clinical implications of an extended-infusion dosing strategy. Clin Infect Dis 2007;44:357-63.

10. Moise-Broder PA, Forrest A, Birmingham MC, et al. Pharmacodynamics of vancomycin and other antimicrobials in patients with Staphylococcus aureus lower respiratory tract infections. Clin Pharmacokinet 2004;43:925-42.

11. Wysocki M, Delatour F, Faurisson F, et al. Continuous versus intermittent infusion of vancomycin in severe Staphylococcal infections: prospective multicenter randomized study. Antimicrob Agents Chemother 2001;45:2460-7.

12. Cataldo MA, Tacconelli E, Grilli E, et al. Continuous versus intermittent infusion of vancomycin for the treatment of Grampositive infections: systematic review and meta-analysis. $J$ Antimicrob Chemother 2012;67:17-24.

13. Rello J, Sole-Violan J, Sa-Borges M, et al. Pneumonia caused by oxacillin-resistant Staphylococcus aureus treated with glycopeptides. Crit Care Med 2005;33:1983-7.

14. Gwee A, Cranswick N, Metz D, et al. Neonatal vancomycin continuous infusion: still a confusion? Pediatr Infect Dis $J$ 2014;33:600-5.

15. Pawlotsky F, Thomas A, Kergueris MF, et al. Constant rate infusion of vancomycin in premature neonates: a new dosage schedule. $\mathrm{Br} J$ Clin Pharmacol 1998;46:163-7.

16. Plan O, Cambonie G, Barbotte E, et al. Continuous-infusion vancomycin therapy for preterm neonates with suspected or documented Gram-positive infections: a new dosage schedule. Arch Dis Child Fetal Neonatal Ed 2008;93:F418-21.
17. Oudin C, Vialet R, Boulamery A, et al. Vancomycin prescription in neonates and young infants: toward a simplified dosage. Arch Dis Child Fetal Neonatal Ed 2011;96:F365-70.

18. Zhao W, Lopez E, Biran V, et al. Vancomycin continuous infusion in neonates: dosing optimisation and therapeutic drug monitoring. Arch Dis Child 2013;98:449-53.

19. Patel $A D$, Anand $D$, Lucas $C$, et al. Intermittent versus continuous infusion of vancomycin in neonates. Arch Dis Child 2012;97:e20.1.

20. Victorian Maternity and Newborn Clinical Network. Neonatal eHandbook - normal laboratory values for neonates. Secondary Neonatal eHandbook - normal laboratory values for neonates. https://www2.health.vic.gov.au/hospitals-and-health-services/ patient-care/perinatal-reproductive/neonatal-ehandbook/pathology/ normal-laboratory-values

21. The Royal Pharmaceutical Society of Great Britain. British national formulary for children 2011-2012. London, UK: The Royal Pharmaceutical Society of Great Britain.

22. Eiland LS, English TM, Eiland EH. Assessment of vancomycin dosing and subsequent serum concentrations in pediatric patients. Ann Pharmacother 2011;45:582-9.

23. Goutelle S, Neely M, Bleyzac N. Comment: assessment of vancomycin dosing and subsequent serum concentrations in pediatric patients. Ann Pharmacother 2011;45:1171-2.

24. Turner L, Shamseer L, Altman DG, et al. Consolidated standards of reporting trials (CONSORT) and the completeness of reporting of randomised controlled trials (RCTs) published in medical journals. Cochrane Database Syst Rev 2012;11:MR000030.

25. Howie SR. Blood sample volumes in child health research: review of safe limits. Bull World Health Organ 2011;89:46-53.

26. Schwartz GJ, Work DF. Measurement and estimation of GFR in children and adolescents. Clin J Am Soc Nephrol 2009;4:1832-43.

27. Hsu DI, Hidayat LK, Quist R, et al. Comparison of methodspecific vancomycin minimum inhibitory concentration values and their predictability for treatment outcome of meticillin-resistant Staphylococcus aureus (MRSA) infections. Int J Antimicrob Agents 2008;32:378-85. 